Referencia para citar este artículo: Pava-Ripoll, N. A. (2017). Discapacidad y configuración del Capital Emocional (CE): el caso de tres padres. Revista Latinoamericana de Ciencias Sociales, Niñez y Juventud, 15 (2), pp. 951-963. DOI:10.11600/1692715x.1521022062016

\title{
Discapacidad y configuración del Capital Emocional (CE): el caso de tres padres*
}

\author{
Nora ANeth PAVA-RIPOLL * \\ Docente Universidad del Valle, Colombia.
}

\begin{abstract}
Artículo recibido en junio 22 de 2016; artículo aceptado en agosto 1 de 2016 (Eds.)
\end{abstract}
- Resumen (analítico): el objetivo del artículo es mostrar la configuración del CE a partir del análisis de los relatos de vida de tres padres cuyos hijos e hijas presentan discapacidad. Los argumentos de las teorías de los campos sociales de Bourdieu y de la antropología de las emociones planteada por Hochschild aportan al piso teórico del concepto. La metodología utilizada fue el enfoque narrativo-biográfico a través de los relatos de vida. Se concluye que el CE surge desde la ruptura ideológica -de la normalidad-que ocurre en el momento del diagnóstico de discapacidad de sus hijos e hijas, pero se acumula desde la confrontación con la estructura social. Sólo uno de los padres logra adquirir CE, pues cuando las personas viven sus vidas en marcos ideológicos establecidos que no se cuestionan, es imposible acumular CE.

Palabras clave: investigación social, ideología, infancia (Tesauro de Ciencias Sociales de la Unesco).

Palabras clave autora: discapacidad, capital emocional, campo social

\section{Disability and configuration of Emotional Capital (EC): the case of three fathers}

- Abstract (analytical): the aim of the article is to demonstrate the configuration of Emotional Capital (EC) through the analysis of the life stories of three fathers whose children have disabilities. The main components of Bourdieu's social field theory and the anthropology of emotions proposed by Hochschild provide the theoretical framework for this study. The methodology used was the biographical-narrative approach through the collection and analysis of life stories. It is concluded that EC emerges from the ideological break with normality that occurs at the time of diagnosis of the disability of their children, and accumulates through confrontations with social structures. Only one of the fathers succeeds in acquiring EC because when people live their lives in established ideological frameworks that are not questioned it is impossible to accumulate EC.

Key words: social research, ideology, childhood (Unesco Social Sciences Thesaurus).

Author key words: disability, emotional capital, social field.

\section{Incapacidade e configuração do Capital Emocional (CE): o caso de três pais}

- Resumo (analitico): o objetivo do artigo é mostrar a configuração do Capital Emocional (CE) a partir da análise das histórias de vida de três pais cujos filhos têm deficiência. Os argumentos da

Este artículo de investigación científica y tecnológica hace parte de la investigación denominada "El capital emocional: un desafío a la ideología de la normalidad" presentada por la autora para optar por el título de Doctora en Ciencias Sociales, Niñez y Juventud, Universidad de Manizales-Cinde. Realizada entre agosto de 2011 y febrero de 2016. Área del conocimiento: Psicología. Subárea: Psicología (incluye terapias de aprendizaje, habla, visual y otras discapacidades físicas y mentales).

** Profesora Asociada Facultad de Salud de la Universidad del Valle. Fonoaudióloga de la Universidad Católica de Manizales, Magíster en Educación y Desarrollo Humano de la Universidad de Manizales-Cinde (Colombia), Doctora en Ciencias Sociales, Niñez y Juventud. Orcid: 0000-0002-4664-7278. Índice H5: 2. Correo electrónico nora.pava@correounivalle.edu.co 
teoria de campo social de Bourdieu e a antropologia das emoções suscitadas por Hochschild fornecem o piso teórica para este conceito. A metodologia utilizada foi a abordagem narrativa-biográfica através de histórias de vida. Conclui-se que a CE surge da ruptura ideológica de normalidade que ocorre no momento do diagnóstico da deficiência de seus filhos, e acumula-se a partir do confronto com a estrutura social. Apenas um dos pais consegue adquirir CE, porque quando as pessoas vivem suas vidas em quadros ideológicos estabelecidos que não são questionadas, é impossível acumular $C E$.

Palavras-chave: investigação social, ideologia, infância (Tesauro de Ciências Sociais da Unesco). Palavras-chave autores: inabilidade, capital emocional, campo social.

\section{-1. Introducción. -2. Metodología. -3. El capital emocional (CE). -4. EI CE y otros capitales en el campo de la salud. -5. Discusión de resultados. -Lista de referencias.}

\section{Introducción}

Mauricio, John y Manuel son tres padres de niño/as con discapacidad ${ }^{1}$. Sus vidas están atravesadas por una realidad inesperada que cobra particular importancia, especialmente en la época actual en la que el discurso de la reproducción está saturado de elección, control e, incluso, perfección (Landsman, 2009). $\mathrm{Su}$ experiencia paternal ha cambiado, pues afrontan realidades diferentes a las esperadas. Por eso, lo que se muestra en este artículo es un acercamiento a la vida de estos padres, revelada a través de encuentros narrativos que hicieron parte del corpus más amplio que configuró la investigación doctoral. Desde miradas sociológicas y antropológicas, la comprensión del mundo emocional de padres y madres de niño/as con discapacidad permitió argumentar el concepto de Capital Emocional (CE) en la medida en que la experiencia subjetivaque ocurre por un acontecimiento particular, se relaciona con la experiencia social. Entender un poco la vida de estos padres y cómo su experiencia sirve para desarrollar estos argumentos sobre el $\mathrm{CE}$, se constituye en eje fundamental de

1 Según Landsman (2009), la utilización del concepto "niño/as con discapacidad" (children with disability) da relevancia en primera instancia a la condición infantil, es decir, un niño es un niño, sin enfatizar su condición y adjudicándole una de muchas otras características que pueda tener. Por otro lado, el concepto "niño/as discapacitado/as" (disabled children), reconoce que un niño con una deficiencia está primariamente discapacitado por la sociedad más que ser una cualidad intrínseca del niño. Sin ánimo de desvirtuar el uso de un término en función del otro, en este documento me referiré a "niños y niñas con discapacidad", no con la idea de entrar en discusiones sobre el tema, asunto que sería pertinente en otras instancias, ni para ubicar ciertas posturas o pensamientos particulares, sino más bien, en coherencia con la normativa colombiana que se refiere a "personas con discapacidad" (PcD). este artículo, especialmente porque uno de los eventos de esa experiencia paternal, se relacionan con una ruptura de la ideología -de la normalidad- que necesariamente los hace reflexionar sobre los sistemas de valores $\mathrm{y}$ creencias, el proyecto futuro e, incluso, su participación en la sociedad.

En los siguientes párrafos de esta introducción se presentan brevemente dos de los argumentos teóricos que fundamentan el CE. Por un lado, la postura sociológica de Bourdieu reconoce la importancia de los capitales en la interacción cotidiana con los diferentes campos en los que padres, madres e hijo/as se desenvuelven; y por el otro, argumentos desde la antropología de las emociones planteados por Hochschild en los que se asume una construcción social de las emociones.

En su fundamentación desde el constructivismo estructuralista, Bourdieu reconoce la presencia de unas estructuras sociales objetivas, que no dependen de la conciencia y la voluntad de los agentes, pero son capaces de constreñir sus prácticas. Para él, el mundo social puede ser representado en un "espacio de múltiples dimensiones, construido sobre la base de los principios de diferenciación o distribución constituido por el conjunto de propiedades que actúan en un universo social concreto" (Bourdieu, 1984/1990, pp. 282-283). El conjunto de propiedades que allí actúan, confieren fuerza y poder a quien detenta dichas propiedades y están representadas por unas posiciones relativas de los agentes distribuidos en ese espacio según el volumen global de capital que poseen y la composición de ese capital. Estas relaciones de poder dan lugar a 
los campos (académico, político, económico, etc.), los cuales no son estáticos, pues allí los agentes ejercen oposición, lucha, fuerzas de poder por determinados tipos de capital que pueden conservar o transformar la estructura social.

Bourdieu define el concepto de capital mediante los recursos, bienes y valores de que disponen las personas que ocupan diferentes posiciones en varios campos. Para él, el capital es "una fuerza dentro de un campo; trabajo acumulado, bien en forma de materia, bien en forma interiorizada o "incorporada" (Bourdieu, 2000). Se refiere a él como la "energía social", una especie de poder para ejercer control, un "instrumento de apropiación de las oportunidades teóricamente ofrecidas a todos" (Bourdieu, 1980/2007, p. 109). El capital permite expresar todas las habilidades y conocimientos a través de los cuales el habitus interactúa en el campo específico donde se expresa socialmente y en donde se da vida a una forma de interés.

El capital económico, es reconocido socialmente como capital, es decir, como medio para ejercer el poder sobre recursos o personas (apropiación de bienes y servicios), sin necesidad de ocultar esta dominación para que sea legítima (Martínez, 2011) (por ejemplo, la herencia o la fortuna alcanzada). El capital cultural puede presentarse en tres formas: incorporado a las disposiciones mentales (invertir en este tipo de capital implica hacer acopio de procesos formativos sobre los cuales se hacen renuncias y privaciones), objetivado (expresado en forma de bienes culturales: pinturas, monumentos, objetos de valor) e institucionalizado (reconocido por las instituciones políticas: títulos académicos). El capital social es el agregado de los recursos actuales o potenciales de que se dispone por pertenecer a un grupo (Bourdieu, 1986); no es independiente de los demás capitales y depende también de un esfuerzo continuado. El capital simbólico es cualquier tipo de capital percibido por agentes sociales cuyas categorías de percepción son de tal naturaleza que les permiten conocerla (distinguirla) y reconocerla, conferirle algún valor (Bourdieu, 1994/1997), por ejemplo, legitimidad, autoridad y prestigio.
Además, Shilling (2012) ha referido un concepto relacionado con 'capital corporal'2 y examina cómo los sujetos identifican el cuerpo como una forma de capital corporal, un poseedor del poder, estatus y formas distintivas simbólicas relacionadas con la acumulación de los recursos. La acumulación de capital corporal se refiere al desarrollo del cuerpo en formas reconocidas como valor poseído en los campos sociales, como tener una buena figura, ser fuerte, bonito, hábil, que a la vez le permiten convertir ese capital corporal en ocio y participación física en el trabajo (Shilling, 2012). Por su parte Scribano (2007) se refiere a este capital corporal en relación con las condiciones de existencia alojadas en el cuerpo del individuo, en el cuerpo subjetivo y en el social.

Por otro lado, es posible afirmar que el discurso de lo emocional se ha posicionado en las últimas décadas e interpela hoy cualquier realidad. Si su sustrato es biológico o cultural, particular o universal, individual o social, si corresponde a la vida privada o pública es parte del sinnúmero de discusiones que aún hoy continúan vigentes y aportan un nuevo matiz a la comprensión de un ser humano emocionalmente constituido. Por ello posturas antropológicas de las emociones como la de Hochschild (1979, 1983, 2008, 2009) también fundamentan el CE.

Hochschild expresa la relación entre emociones y estructura social en la que muestra la dinámica de un mundo emocional privado hacia el mundo socio-laboral (Hochschild, 1983). Según la autora hay una serie de normas sociales que se aplican a comportamientos emocionales esperados ante ciertas situaciones; por ejemplo se espera que las personas estén felices en los matrimonios y tristes en los funerales; además está previsto un tiempo prudencial para sentirse afligido y "llorar" la muerte de un ser querido. Este comportamiento acordea estas circunstancias es loqueHochschild plantea como "normas emocionales" o "reglas del sentimiento" (feeling rules), es decir el

2 El término en inglés se expresa 'Physical capital', aunque algunos autores lo traducen como capital físico; yo me referiré a él como capital corporal en relación al cuerpo físico, pero también a las capacidades mentales. 
comportamiento interno que nos prepara para actuar externamente, constituyendo un modelo de control social que impulsa una actuación emocional acorde a determinadas circunstancias sociales, indicando el sentimiento apropiado para cada caso.

Hochschild (1979) se refiere al concepto de "trabajo emocional" (emotional work o emotion managment) como las "acciones orientadas a tratar de cambiar en algún grado o calidad una emoción" (p. 561). Es decir, no hace énfasis en el producto sino en el esfuerzo, la gestión, en el acto de tratar de modificar las emociones, independientemente de si se tiene éxito o no. Este concepto es diferente al de control emocional, el que es sugerido para contener o prevenir los sentimientos, tiene que ver también con evocación o estimulación de ciertos sentimientos deseables, pero inicialmente ausentes. Se refiere a un cambio real de los sentimientos, no solo de la expresión de estos, lo que puede hacerse por varios métodos. La autora diferencia emotional work de emotional labor en la transición del ámbito de lo privado (emotional work) a lo público (emotional labor). Es decir, el conjunto de normas emocionales que operan en nuestra vida privada son diferentes en el ámbito laboral mediado por transacciones económicas.

La obra de Hochschild ha tenido gran desarrollo en la esfera organizacional ya que el "trabajo emocional" ha sido muy útil para el desarrollo de investigaciones que establecen las relaciones entre prestación de servicios, economía y bienestar de los trabajadores. En aras de dejar de lado el interés sobre el contexto laboral, en adelante se hará referencia a Esfuerzo Emocional (EE) para establecer una relación entre emotional work y emotional labor, puesto que se trata del trabajo emocional de los padres y las madres desde el ámbito privado -emotional work- (relaciones con la pareja, demás miembros de la familia) hacia el público -emotional labor- (los médicos, terapeutas, profesores, etc.) en el que se establecen negociaciones, transacciones en beneficio de ellos mismos y de sus hijos, pero sin que necesariamente medie una relación de índole laboral en una organización que tiene establecidas unas normas de comportamiento sobre el manejo de las emociones. Es decir, es viable que existan transacciones económicas que orienten la prestación de un servicio, como por ejemplo pagar por el cuidado del hijo/a, o contratar determinados médicos o terapeutas, o matricularlo en determinados colegios. Sin embargo, en ausencia de códigos laborales que indiquen como debe establecerse la relación emocional, en estas transacciones no se expresa la rigurosidad del concepto emotional labor.

\section{Metodología}

Variados son los acercamientos a los enfoques narrativo-biográficos, entre ellos, historias y relatos de vida. Solo para mencionar algunos, Mallimaci y Giménez (2006) afirman que la historia de vida "es el estudio de un individuo o familia, y de su experiencia de largo plazo, contada a un investigador y/o surgida del trabajo de documentos o registros vitales" (p. 178); Pujadas (2000) la define como un "relato exhaustivo autobiográfico obtenido por el investigador mediante entrevistas sucesivas en las que el objetivo es mostrar el testimonio subjetivo de una persona en la que se recogen tanto acontecimientos como valoraciones que esa persona hace de su experiencia" (p. 132). Es claro también que desde diferentes disciplinas, han sido variados los términos para referirse a las diferentes modalidades del enfoque narrativo-biográfico, lo que ha hecho que hoy en día se presenten una variedad de conceptos y términos, en ocasiones redundantes y confusos, que pueden complejizar su comprensión y uso. Algunos de los más usados son: biografía, historia de vida, autobiografía, fuente oral, historia oral, relato biográfico. Bolivar y Domíngo (2006) distinguen entre Life story, récit de vie, narración, relato de vida como la narración de una vida tal como la persona la ha vivido y/o cuenta; y Life history, histoire de vie, historia de vida, el conjunto del anterior concepto y las elaboraciones externas de biógrafos o investigadores, así como los registros, entrevistas, etc., que permiten validar esta narración o esta historia.

Los resultados que se presentan en este artículo hacen parte de una investigación doctoral "el capital emocional: un desafío a 
la ideología de la normalidad". Este estudio se desarrolló en la ciudad de Manizales, Colombia desde el año 2012 hasta el 2015; la población que participó fueron padres, madres y cuidadores de niños y niñas entre 0 y 12 años que tuvieran algún tipo de discapacidad, con quienes se recolectó información a través de tres corpus en los que ese utilizaron técnicas como: corpus 1: entrevistas estructuradas y talleres de narrativas conversacionales (PavaRipoll, 2015), corpus 2: entrevistas narrativas y corpus 3: observaciones estructuradas y participativas. Para efectos de este artículo se presentarán parte de los resultados obtenidos a través del corpus 2 en el que se trabajó con 13 madres y 7 padres. Teniendo en cuenta estos aspectos, esta investigación tomó elementos de los relatos de vida, puesto que se centró en el estudio de uno o varios individuos y recopiló datos a través de sus experiencias individuales. El eje central fueron las voces de ellos mismos, pues cada persona vive su realidad desde sus propias versiones y desde sus propias visiones y cuando se trata de las emociones, de lo que se siente ante una situación vivida, los testimonios de quienes viven esa realidad tienen un significado emocional particular. Sin embargo, es de aclarar que por la complejidad de todo el proceso investigativo realizado, en este artículo se muestra solo una parte de estos relatos.

Las entrevistas narrativas se realizaron a través de variados encuentros individuales con ellos. Cada entrevista fue registrada en audio, con algunas notas de campo para enfatizar algunos aspectos considerados importantes para revisar más adelante o profundizar en la misma entrevista. La relación con los entrevistados fue de colaboración, teniendo en cuenta que "la investigación narrativa es un proceso de colaboración que conlleva una mutua explicación y re-explicación de historias a medida que la investigación avanza" (Connelly \& Clandinin, 1996). Cuando se trataba de una segunda o tercera entrevista con la misma persona, inicialmente se ampliaba y modificaba aspectos de la primera entrevista, cuidando no ser reiterativa. Además se realizaban preguntas sobre situaciones que habían quedado pendientes de resolver (una cita o un examen médico importante, la búsqueda de colegio para su hijo, etc.), para lo cual se retomaban aspectos de la(s) entrevista(s) anterior(es), resaltando los aspectos que eran necesarios ampliar o aclarar.

En el análisis, realizado a la par que la recolección de los datos, se organizó la información por núcleos temáticos, agrupando la información en grandes temas que, aunque no siempre eran comunes a todos los relatos, vislumbraron una tendencia en ellos. Esto permitió visualizar la complejidad de las interacciones entre el mundo social y subjetivo para dar cuenta de las configuraciones de sentido construidas. En este artículo se presenta una de las categorías configuradas a partir de sus experiencias de vida en torno a la rehabilitación y el campo de la salud. Los siguientes dos apartados muestran el análisis de los resultados y la discusión teórica sobre esta categoría.

\section{El capital emocional (CE)}

El CE se refiere al conjunto de emociones y experiencias que definen las maneras cómo las personas se constituyen subjetivamente, así como de inter-actuar, interpretar, expresar y vivir los acontecimientos de la realidad. El $\mathrm{CE}$ define cómo asume cada agente social los acontecimientos y les otorga la posibilidad de actuar reflexivamente. Este actuar reflexivo es logrado por la interacción sentimiento-razón en una práctica social determinada en las que los agentes se dan cuenta de que las maneras de sentir y de verse afectados por las circunstancias de vida, no sucede solamente por ese hecho en sí (en este caso tener un hijo con discapacidad), sino que se produce por vivir esa circunstancia en una realidad que restringe las posibilidades para vivirlo de otra manera. Por ello el CE es una movilización de adentro hacia afuera-subjetiva-, que permite entablar diversas formas de relación con el entorno y de conectarse con el mundo. Más que tratarse de medidas fisiológicas, o ser visible corporalmente, o más allá de las manifestaciones que pueden caracterizarse como negativas o positivas, o del éxito esperado frente a sentimientos y emociones controladas, el capital emocional surge en el intento (de reflexividad/confrontación/de-construcción), fallido o no, mediante la cual asumimos una posición activa sobre nuestro ser emocional. 
Esa experiencia subjetiva que ocurre por un acontecimiento es la que va a relacionarse con la experiencia social. El CE le concede a los agentes la disposición para tomar ciertas decisiones sobre su cotidianidad, para actuar de determinadas formas, por ello emerge y se fundamenta en las formas en que los padres y las madres resuelven sus problemas prácticos y es particular a cada agente social, según su posición en el campo, en este caso, el campo de la salud.

Los resultados de este estudio muestran que las personas de cualquier estrato social, raza o género, poseen los elementos necesarios para garantizar que en circunstancias particulares pueda lograrse la amalgama que hará emerger y potenciar el CE. Además, el CE de los padres de niño/as con discapacidad se acumula en mayor medida a partir de la ruptura ideológica -de la normalidad- que ocurre en el momento del diagnóstico de la discapacidad de sus hijo/as. Esto se puede comprender pues al paradigma médico - rehabilitador que ha dominado la mirada de la discapacidad durante muchos siglos, subyace una ideología de la normalidad acorde a un sistema de producción y reproducción social. Esta ruptura ideológica se constituye en manantial para el $\mathrm{CE}$, es el gatillo que permite que cada agente reconozca y haga conciencia de su presencia latente y que empiece a hacer parte de la disputa dentro del campo.

El CE es particular a cada agente social según su posición en el campo, por lo que empieza a fluir y a interactuar estratégicamente con los demás capitales que posee cada uno; sin embargo su acumulación toma tiempo y esfuerzo, especialmente por las exigencias sociales vigentes sobre la infancia y las expectativas de normalidad de los padres frente a sus hijos. A pesar de que las situaciones de crisis y choque emocional lo apalancan, cada agente, según su trayectoria individual vive su experiencia de manera diferente, según su confrontación con la estructura social. Esto hace posible entender por qué en la obra de Bourdieu campo y capital no son comprensiones aisladas en tanto que el valor de los capitales es relativo dependiendo del campo donde los agentes se desenvuelven.

\section{El capital emocional y los otros capitales en el campo de la salud.}

En la articulación del capital emocional con otros capitales se esbozan unas variaciones de la realidad que viven los padres cuyos niño/ as tienen una discapacidad. Esto se debe a sus posibilidades de acceso y al tipo de afiliación al sistema de salud. A la vez, esta realidad es muy cambiante en todos los países del mundo, por lo que se describirá lo que sucede en el caso colombiano. Desde 1993 la ley 100 planteó un Sistema General de Seguridad Social en Salud (SGSSS) cuyo propósito fue alcanzar la cobertura universal en la prestación de los servicios de salud desde tres regímenes: contributivo, subsidiado y vinculado. En el régimen contributivo se encuentran las personas que tienen una vinculación laboral, es decir, con capacidad de pago como los trabajadores formales e independientes y los pensionados. El régimen subsidiado es el mecanismo mediante el cual la población sin capacidad de pago tiene acceso a los servicios de salud a través de un subsidio que ofrece el Estado. En el régimen vinculado se encuentra la población pobre o vulnerable que no tienen afiliación al Sistema de Salud en alguno de los dos regímenes anteriores (República de Colombia, 2010).

En este sentido, es claro cómo en Colombia, el sistema de salud está fragmentado según la capacidad de pago de las personas, es decir está ligado al ingreso económico; hay "atención para pobres, para trabajadores formales y para ricos, con serias diferencias en oportunidad y calidad" (Hernández, 2000, p. 121). La atención de las personas en los distintos regímenes se hace a través de las Entidades Promotoras de Salud (EPS) quienes administran el riesgo de salud de sus afiliados y garantizan la prestación de los servicios. También existen en el mercado de la salud los planes de medicina prepagada que ofrecen un servicio privado de salud según la capacidad de pago del usuario (Según un artículo en la revista Portafolio de julio 7 de 2015, la Asociación Colombiana de Empresas de Medicina Integral -Acemi-, asegura que hay cerca de 250.000 colombianos afiliados a los planes complementarios y alrededor de un millón a medicina prepagada). Por esto es 
posible confrontar las experiencias que los padres relatan, de acuerdo a las diversas formas de vinculación al sistema de salud, lo que a la vez propicia formas diversas de interacción en el campo de la salud.

Esas formas de vinculación al sistema de salud, hacen que padres y madres empiecen a actuar de determinadas maneras, especialmente frente a la toma de decisiones sobre lo que consideran más beneficiosos para su hijo/a con discapacidad, iniciando así un proceso que tiene en la base determinados saberes. El hecho de acudir a ciertos profesionales, implica una serie de transacciones no sólo a partir de su capital económico, sino de sus conocimientos cotidianos sobre las necesidades de sus hijo/as con discapacidad y los saberes que demuestran tener estos profesionales (Venturiello, 2012).
Para poder analizar esta interrelación de fuerzas y capitales, se muestran los casos de tres padres: Mauricio (papá de Teo), John (de Jero) y Manuel (de Abi), desde la perspectiva de sus capitales personales. Se aclara que el capital corporal de los tres es considerado como alto, pues poseen cuerpos sanos, acorde a las expectativas sociales que les permite desempeñarse de manera esperada a nivel corporal. Este capital, a diferencia de los demás, no está en disputa. Sin embargo, el capital corporal de los hijo/as sí marca ciertas diferencias. Solo para orientar un poco al lector, a continuación se describe brevemente los capitales corporales de los niño/as a través de algunas características, físicas, lingüísticas y cognitivas de cada uno.

Tabla 1: Capitales corporales de Teo, Jero y Abi.

\begin{tabular}{|c|c|c|l|c|}
\hline Nombre & $\begin{array}{c}\text { Edad } \\
\text { (años) }\end{array}$ & Diagnóstico & $\begin{array}{c}\text { Capital } \\
\text { Corporal }\end{array}$ \\
\hline Teo & 4 & $\begin{array}{c}\text { Descripción } \\
\text { psicomotor, } \\
\text { debido a } \\
\text { alteración } \\
\text { genética. }\end{array}$ & $\begin{array}{l}\text { Teo se desplaza en ambos pies sin dificultad, aunque le cuesta } \\
\text { llevar a cabo otras actividades motoras más complejas como } \\
\text { saltar o trepar. Su expresión lingǘstica es limitada, se expresa con } \\
\text { frases cortas pero posee una excelente comprensión del lenguaje } \\
\text { oral. Son evidentes en él los trastornos del comportamiento, } \\
\text { fácilmente hace pataletas en casa, calle y colegio. Está } \\
\text { escolarizado en colegio privado. }\end{array}$ \\
\hline Jero & 10 & $\begin{array}{c}\text { Parálisis } \\
\text { cerebral } \\
\text { espástica }\end{array}$ & $\begin{array}{l}\text { Tiene limitaciones para su autonomía personal debido a su } \\
\text { parálisis cerebral. No camina, mantiene su postura sentado con } \\
\text { apoyos. Posee un lenguaje muy funcional que le permite entablar } \\
\text { conversaciones. Está escolarizado en colegio semi-privado }\end{array}$ \\
\hline Abi & 3 & $\begin{array}{c}\text { Parálisis } \\
\text { cerebral } \\
\text { espástica. }\end{array}$ & $\begin{array}{l}\text { A nivel motor, Abi no sostiene cabeza ni se mantiene sentada, ni } \\
\text { tampoco posee marcha. Lingüísticamente no realiza expresiones } \\
\text { verbales aunque reconoce las voces de su padre y su madre y } \\
\text { responde a ellas. No está escolarizada }\end{array}$ \\
\hline
\end{tabular}

A continuación se describen las situaciones particulares de cada uno de estos padres para poder dar cuenta de sus capitales.

Mauricio, el papá de Teo es empresario, posee altos ingresos (tiene un capital económico alto), que le permite un buen nivel de vida como tener casa en uno de los mejores barrios de la ciudad, viajes, ser socio de clubes privados (capital cultural y social también alto). Además posee una formación a nivel profesional, por lo que su capital cultural incorporado a manera de títulos académicos le ha permitido acrecentar su capital económico. Por el éxito que ha logrado en su empresa tiene un reconocimiento profesional y social también alto, dado además por el hecho de que él hizo su fortuna estando antes en otras posiciones en el campo (capital simbólico). A pesar de que Mauricio está afiliado al sistema de salud (régimen contributivo) y su hijo es beneficiario de éste, afirma nunca haberlo usado para su hijo. Todos los costos los ha asumido él, de su propio bolsillo o a través del seguro médico privado y sin necesidad de instaurar acción de tutela como mecanismo de 
defensa del derecho a la salud de su hijo. En los diferentes espacios donde Teo se desempeña de manera cotidiana, como el colegio y las terapias, cuenta con una acompañante, quien es una persona con título profesional y quien incluso fue traída de otro país de Latinoamérica. Por todo esto, se observa que Mauricio, por su alto capital económico, tiene la posibilidad de hacer grandes inversiones en la rehabilitación de su hijo.

Yo personalmente nunca uso la EPS y yo creo que con Teo tampoco. Inclusive con lo de Teletón nos decían que con la EPS tenían la obligación de darnos una atención para Teo, pero no [...]. He tenido la posibilidad de llevarlo a medicina particularmente o medicina prepagada, siempre me lo han atendido muy bien. [...]. Todo siempre ha sido particular (Mauricio).

El relato de Mauricio demuestra que por su posición privilegiada otorgada por su alto capital económico ha podido dar a su hijo una atención también privilegiada al tener la posibilidad de llevarlo a los médicos y especialistas seleccionados cuidadosamente $\mathrm{y}$ no de una lista restringida, como sucede en el caso de los regímenes contributivo y subsidiado. Además afirma:

La inversión económica que se hace con él (Teo) es muy grande y yo pienso que la discapacidad con pobreza es dejar al niño ahí tirado a lo que él pudiera hacer. Realmente pienso que Teo ha logrado avanzar mucho porque se han tenido los medios para hacerle lo que nos van diciendo que es necesario, sin escatimar recursos [...]. Sé que me toca trabajar duro pero sé que estoy haciendo todo por mi hijo. Por ejemplo yo tengo una profesional del exterior que la traje para trabajar con Teo a la que le pago $\$ 7.000 .000$ (aproximadamente 2.300 US) mensuales, le pago su apartamento, y no más en ese costo ya van casi $\$ 9.000 .000$ más los otros tratamientos y terapias. Yo diría que solo en Teo se van mensuales unos $\$ 10.000 .000$ más la acompañante en el colegio. Entonces yo entiendo que para la sociedad, para el común de las personas disponer de ese dinero para poder atender a su hijo no puede ser tan fácil (Mauricio).

Él reconoce su posición privilegiada desde su alto capital económico al poder costear todo esto. Se observa como las clases altas son regularmente las beneficiadas de las desigualdades, pues entre más alta sea, más difícil es para las personas aceptar que su capital económico no va a lograr resolver la discapacidad de su hijo, como en el caso de Mauricio.

John, el papá de Jero, es empleado y posee ingresos medios. De hecho es auditor médico de una EPS, lo que implica que tiene un conocimiento mayor que los demás sobre el funcionamiento del sistema de salud, lo cual se considera una ganancia en términos de capital cultural, incluso capital social. Sus ingresos económicos no son tan altos como los de Mauricio. Es decir, de John se podría decir que no posee tanto capital económico como Mauricio, pero en su actuación en el campo posee más capital cultural y social que él, pues la posición privilegiada que ocupa como médico ha significado ganancia de estos dos capitales, lo que se evidencia en este relato:

Yo diría que Jero está vivo porque es hijo $\mathrm{de}^{3}$. Y porque hicimos todo el acompañamiento. Porque las expectativas de vida eran muy pocas y la capacidad de que viviera escasa. Vimos morir muchos niños con lesiones menores que Jero en la UCI; y yo no creo que por los profesionales, sino que uno estaba más pendiente y lograba más empatía con los profesionales, y estaba ahí con todas las cosas, y tratábamos de apoyar en todo lo que se pudiera. Eso hacía que las cosas fluyeran. Yo creo que desde el primer momento que Jero nace y sale adelante a pesar de sus muchas enfermedades, lo hizo porque éramos profesionales, pues porque nosotros estábamos ahí, y porque había mucho acompañamiento

3 La narrativa de John muestra aquí el punto al final de la frase. Él se refiere a que él es médico y fue gracias a su profesión que logró 'sacar adelante' a su hijo. 
de los colegas. Estoy casi convencido que si hubiera sido otro niño, no hubiera salido adelante. Poco probable, porque se requerían muchas cosas, mucho apoyo y era la época del hospital en crisis. Tocaba hacer muchas cosas para que el niño estuviera bien y tuviera algún grado de probabilidad de sobrevivir al evento (John).

Estas ganancias de capitales (cultural y social, más que el económico) le permitieron acceder a beneficios para su hijo como estudios diagnósticos complejos y acceso a médicos especialistas reconocidos, sólo que la inversión económica que ha hecho para ello ha sido menor a la de Mauricio, pues entre la EPS y la medicina prepagada se han subsidiado estos costos. Jero también tiene una acompañante tiempo completo, con título de técnico pues es auxiliar de enfermería, quien no es pagada por su padre, sino que es pagada a través del sistema de salud. Además el capital cultural y social de John le permite ser crítico con el sistema de salud del que hace parte:

El sistema de salud es absolutamente excluyente para estos niños, apenas estamos logrando los primeros pinitos con los procesos de rehabilitación; el sistema de salud está en una concepción de atender terapia física pero 6 pacientes por terapeuta y sesión. Y estos muchachos necesitan un terapeuta, una hora completa para uno solo, no puede ser para dos. La terapia ocupacional está diseñada para tres muchachos con déficit de atención y ponerlos a trabajar entre ellos, lo cual tampoco funciona con ellos. La terapia del lenguaje porque contamos con una persona súper especializada que logró cosas. Porque él presentó lo que presentan todos los muchachos, babeo, sensibilidad en la boca, falta de deglución y eso no se logra en los sistemas de salud ipara qué! Desde la perspectiva que tiene un niño aun con tutela, para lograr una rehabilitación como la de Jero, -que no es total-, se requiere lo mejor. La gente es buscando aparatos si no caminan por sí solos. $\mathrm{Si}$ usted va a un centro de neurodesarrollo no hay aparatos, muchas camillas, pero no aparatos (John).

Manuel, el papá de Abi, también es empleado, pero con ingresos menores (se puede afirmar que tiene menor capital económico que los dos padres anteriores); realizó estudios secundarios y actualmente se encuentra adelantando estudios universitarios (trabaja y estudia de noche), o sea que también posee menos capital cultural incorporado e institucionalizado que ellos. Su afiliación al sistema de salud está dada desde su vinculación laboral -régimen contributivo-, pero a diferencia de Mauricio y de John, no posee otros planes médicos alternos. Abi no cuenta con acompañante, ni ha podido adaptársele una silla o coche con medidas acordes a su edad y que favorezca su proceso de [re]habilitación y a la vez les dé la posibilidad a los padres de acceder más frecuentemente a otros entornos. De hecho, Abi no está escolarizada. Al contrario de John, Manuel expresa su agradecimiento con el sistema de salud.

Bueno, no, pues lo que es EPS, especialistas, terapeutas, ahí si lo contrario, el apoyo ha sido bastante bueno. La EPS nos ha brindado todos los servicios que se han pedido. Y por parte de las terapeutas ha sido el proceso que ellos están llevando con la niña y la evolución que le han visto. El apoyo que ellos nos han dado ha sido bastante grande. $\mathrm{O}$ sea con respecto a eso, agradecidos. Ahí sí, respecto a los servicios que nos prestan, todo bien, nada negativo, todo muy bien. La EPS nos ha corrido con todo, pero así que yo diga dificultades o alguna cosa que nosotros pensemos que nos falta algo, no. O sea, hasta el momento nos han corrido con todo y no hemos tenido así nada (Manuel).

Los capitales de Mauricio, John y Manuel se describen en la Tabla 2. 
Tabla 2: Características de los capitales de tres padres en el campo de la salud.

\begin{tabular}{|c|c|c|c|c|c|}
\hline Padre & $\begin{array}{c}\text { Capital } \\
\text { Económico }\end{array}$ & $\begin{array}{c}\text { Capital } \\
\text { Cultural }\end{array}$ & Capital Social & $\begin{array}{c}\text { Capital } \\
\text { Simbólico }\end{array}$ & $\begin{array}{c}\text { Capital } \\
\text { Corporal }\end{array}$ \\
\hline Mauricio & Alto & Alto & Alto & Medio & Alto \\
\hline John & Medio & Alto & Alto & Alto & Alto \\
\hline Manuel & Bajo & Medio & Bajo & Bajo & Alto \\
\hline
\end{tabular}

Pero se hará referencia ahora al CE. Si la ruptura de la ideología de la normalidad es su disparador, John y Manuel vivieron este proceso de manera vertiginosa desde el nacimiento o los primeros meses de vida de sus hijos, debido a la inmediatez relacionada con la supervivencia que estaba en juego. El padre de Teo, vivió este proceso de manera gradual, pausada, viendo cómo 'algo' en el desarrollo de su hijo 'no estaba bien', hasta que a los 30 meses de edad recibió el diagnóstico genético que confirmó la discapacidad del niño. La ruptura de la ideología de la normalidad hizo que sus creencias y expectativas se pusieran en tela de juicio, sus prácticas sociales cotidianas se modificaran, pensaran las cosas de manera diferente pues sus hijos ya no iban a funcionar como 'deberían' funcionar. Esto hace que la potencia del capital emocional asuma diferentes dimensiones para cada uno de los agentes.

El esfuerzo emocional (EE) favorece la acumulación de este capital, pues éste se evidencia en la tensión entre lo que 'quieren' y 'deben' sentir, es decir, el sentimiento 'correcto' que deberían experimentar. La norma social se relaciona con las reglas del sentimiento. Así por ejemplo bajo el manto de 'es mi hijo y hago todo lo que esté a mi alcance por darle lo mejor' se evidencia un EE.

En necesario hacer una distinción entre captar una regla del sentimiento en función de lo que esperamos sentir con relación a la atención en salud de nuestros hijos y lo que deberíamos sentir. Por ejemplo, Manuel el papá de Abi puede tener la expectativa realista de que no recibirá la atención en salud adecuada que requiere su hija, no sólo por la complejidad del caso, sino por sus condiciones económicas y sociales; sin embargo se siente agradecido por la atención que recibe. En otras palabras, cuando Manuel expresa su agradecimiento y refiere 'nada negativo, todo muy bien' puede estar comparando con una expectativa idealizada sobre lo que quiere darle a su hija y mediar entre la regla del sentimiento y la elaboración emocional, lo que equivaldría a 'quisiera no necesitar nada de eso, pero ya que debo acceder a esos servicios por el bien de la niña, debo sentirme agradecido de que se los den'. Además cuando Manuel manifiesta 'el apoyo que ellos (la EPS) nos han dado ha sido bastante grande', reconoce una negación de las responsabilidades del Estado, la cual pasa a ser de ellos como padres. El pronombre 'nos' implica que 'esto lo debo asumir yo -o nosotros como padres', los demás (en este caso el sistema de salud) 'apoyan'. De hecho, en otro relato él reconoce que debe prepararse más, estudiar (adquirir mayor capital cultural) para mejorar las condiciones de vida de su familia (convertirlo en capital económico).

Por otro lado Mauricio el papá de Teo, quien ha usado siempre la medicina privada para la atención de su hijo, puede tener una acompañante (profesional) pagado por él, acceder a exámenes diagnósticos complejos, buscar cada vez nuevos recursos terapéuticos y de rehabilitación que han posibilitado incluso viajar a otro país para acceder a otras terapias especializadas. John, médico, reconoce un sistema de salud excluyente hacia los niños/as con discapacidad y que lo que se logra es fruto de saber moverse hábilmente en el campo. Así por ejemplo Jero, al igual que Teo, tiene una auxiliar de enfermería que lo acompaña todo el día en la figura de "sombra" o "imagen en espejo" que le permite resolver asuntos propios de su salud y restricciones de autonomía por su discapacidad.

Pero a diferencia de John, quien es bastante crítico con el sistema, Manuel no piensa necesitar nada más cuando afirma 'que nosotros pensemos que nos falta algo, no' a pesar de que Abi no tiene acompañante 
"sombra", ni una silla adaptada especialmente a sus necesidades físicas y, como Jero, tiene un diagnóstico de parálisis cerebral espástica. Cuando John expresa que se requiere lo mejor para rehabilitar a los niños que presentan discapacidad, está refiriéndose no sólo a unas terapias con unas condiciones especiales en cuanto a tiempo y disponibilidad de atención, sino a alta tecnología, la cual es escasa y poco se implementa, y lo ratifica cuando dice 'si usted va a un centro de neurodesarrollo no hay aparatos, muchas camillas, pero no aparatos'. El sabe que hay más cosas que se pudieran conseguir para Jero, lo que evidencia una elaboración de las emociones de tipo evocativo (Hochschild, 2008) pues su expectativa está puesta en algo que desea (la mejor tecnología al alcance de las necesidades de su hijo) pero no posee o posee solo en parte. En los relatos de John no es evidente el uso del pronombre "nos", como en los de Manuel. Él reconoce una gran parte de responsabilidad del Estado en la manera de comprometerse con la discapacidad, en este caso, en la infancia. Los logros obtenidos para la calidad de vida de Jero se deben, no solo a su capital económico, sino a sus gestiones dentro del campo, que le ha implicado un EE.

\section{Discusión de resultados}

A través de los relatos de los padres es posible comprender que el $\mathrm{CE}$ emerge en circunstancias particulares que se presentan en sus vidas y se acumula en la medida en que ellos empiezan a transitar un camino en la búsqueda, no sólo de la ratificación de un diagnóstico, sino de una posible restauración. La riqueza de otros capitales, no necesariamente favorece su adquisición. Aunque el CE se exprese subjetivamente, no es posible obtenerlo de manera individual, necesariamente su acumulación adquiere sentido a partir de la confrontación con las estructuras sociales, en el choque contra el aparato ideológico.

Por ello es posible comprender por qué Mauricio posee bajo CE. Su cuantioso capital económico ha hecho que nunca se haya visto confrontado socialmente; en sus acciones cotidianas no tiene que llegar al límite de sí mismo, ni de sus propias relaciones porque él suple con su dinero lo que el sistema no le provee. Él no tiene la necesidad de entrar en este choque ideológico, ni siquiera de cuestionar repensar, de-construir y mucho menos restaurar la manera en que ha venido reproduciendo esa ideología. Por ello, cuando las personas viven sus vidas en marcos ideológicos establecidos que no se cuestionan, es imposible acumular CE.

Pero no es que la mayor acumulación de CE sea inversamente proporcional al capital económico de los agentes. Pues contrariamente a Mauricio, Manuel, posee poco capital económico e igualmente posee bajo $\mathrm{CE}$. Manuel no se cuestiona el sistema, es más, vive agradecido con él porque le 'ayuda' a sobrellevar una responsabilidad que él considera suya y de su pareja. Las narrativas de Manuel dan cuenta de que sólo por SU agencia personal su hija estará bien y logrará 'hacer cosas'. La mirada siempre puesta en el cuerpo de su hija -en su capital corporal-, en lo que ella debe desarrollar y en los requisitos que debe cumplir, por ejemplo, para acceder a un sistema educativo, le impiden a Manuel ver las estructuras sociales y los aparatos ideológicos que han hecho posible esas comprensiones individualistas. Aunque Abi, su hija, pudiera acceder a otros beneficios que no necesariamente dependen del capital económico de su padre, el esfuerzo emocional que él hace, no es suficiente para hacer esa ruptura ideológica que le permitiría acumular CE.

Podría entonces decirse que es John quien logró generar mayor CE. Este capital se expone en los logros, en la acción, en la reflexión, el bienestar y la tranquilidad que le da haber hecho lo necesario por su hijo, independiente del capital corporal de éste. Aunque no posea tanto capital económico, las interacciones entre los capitales emocional, social y cultural, sobre los que tiene ventaja en este campo respecto a Manuel y Mauricio, le sirven para obtener mejores servicios. Por otro lado, el CE se evidencia en la capacidad de operar políticamente; es decir, entender que en el entramado entre estructura social y sentimientos se construye capacidad de acción política. Esto contraviene las ideas de Bourdieu sobre el capital económico que es el que al 
final va a permitir la apropiación de bienes y recursos. En este caso, por ejemplo, fue a partir de las interacciones de capitales emocional, social y cultural que estos se convirtieron en servicios que le permiten a John dar cuenta de que el tratamiento y la atención que va a recibir su hijo van a ser siempre las mejores, así no posea tanto capital económico.

Se hace entonces preciso comprender que no todas las personas que tienen la posibilidad de sobreponerse a situaciones adversas desarrollan CE. No se trata de una operación matemática de acumulación o disminución de unos capitales en beneficio de otros. En la fusión de ingredientes que tienen que ver con la emoción, la estructura social y la ruptura ideológica, dados en su justa medida, surge la posibilidad de acumular CE.

\section{Lista de referencias}

Bolivar, A., \& Domíngo, J. (2006). La investigación biográfica y narrativa en Iberoamérica: campos de desarrollo y estado actual. Forum: Qualitative Social Research, 7 (4), pp. 131-153.

Bourdieu, P. (1986). The forms of capital. En J. G. Richardson (ed.) Handboodk of Theory and research for the Sociology of Education. New York: Greenwood Press.

Bourdieu, P. (1984/1990). Espacio social y génesis de clase. En P. Bourdieu (ed.) Sociología y cultura. México, D. F.: Grijalbo.

Bourdieu, P. (1994/1997). Razones prácticas. Sobre la teoría de la acción. Barcelona: Anagrama.

Bourdieu, P. (2000). Las formas de capital. Capital económico, capital cultural y capital social. En P. Bourdieu (ed.) Poder, derecho y clases sociales. Bilbao: Desclée de Brouwer.

Bourdieu, P. (1980/2007). El sentido práctico. Buenos Aires: Siglo XXI.

Buitrago-Echeverry, M T. (2013). Discapacidades peregrinas. Construcciones Sociales de la Discapacidad en Colombia: aportes para Salud Pública la desde una Perspectiva Crítica. Doctorado Interfacultades de Salud Pública, Universidad Nacional de
Colombia, Bogotá, D. C., Colombia.

Connelly, M. \& Clandinin, D. J. (1996). Relatos de experiencia e investigación narrativa. En J. Larrosa, R. Arnaus, V. Ferrer, N. Pérez de Lara, M. Connelly \& J. Clan (eds.) Ensayos sobre narrativa y educación. Barcelona: Laertes.

Hernández, M. (2000). El Derecho a la Salud en Colombia: obstáculos estructurales para su realización. Revista Salud Pública., 2 (2), pp. 121-144.

Hochschild, A. (1983). The managed heart. Commercialization of human feelings. Berkeley: University of California Press.

Hochschild, A. (2008). La mercantilización de la vida íntima. Apuntes de la casa y el trabajo. Madrid: Katz.

Hochschild, A. (2009). Introduction: an emotions lens on the world. En D. Hopkins, J. Kleres, H. Flam \& H. Kuzmics (eds.) Theorizing emotions. sociological explorations and applications, (pp. 29-38). Berlín: Campus.

Hochschild, A. (1979). Emotion Work, Feeling Rules, and Social Structure. American Journal of Sociology, 85 (3), pp. 551-575.

Landsman, G. H. (2009). Reconstructing motherhood and disability in the age of "perfect" babies. New York: Routledge Taylos \& Francis Group.

Mallimaci, F. \& Giménez, V. (2006). Historia de vida y métodos biográficos. En I. Vasilachis (ed.) Estrategias de investigación cualitativa. Barcelona: Gedisa.

Martínez, J. (2011). Las clases sociales y el capital en Pierre Bourdieu: un intento de aclaración. Salamanca: Universidad de Salamanca.

Pava-Ripoll, N. A. (2015). Narrativas conversacionales con familias y docentes de niños y niñas con discapacidad: un aporte metodológico. Interdisciplinaria, 32 (2), pp. 203-222.

Pujadas, J. (2000). El método biográfico y los géneros de la memoria. Revista de Antropologia Social, 9, pp. 217-158.

República De Colombia (2010). Encuesta Nacional de Demografia y Salud 2010. Bogotá, D. C.: Ministerio de la Protección Social. 
Scribano, A. (2007). A modo de epílogo. ¿Por qué una mirada sociológica de los cuerpos y las emociones? En R. Luna \& A. Scribano (eds.) Contigo Aprendí... Estudios Sociales de las Emociones. Buenos Aires: CEA, Conicet, Universidad Nacional de Córdoba, Cusch, Universidad de Guadalajara.

Shilling, C. (2012). The Body \& Social Theory. London: Sage Publications.

Venturiello, M. P. (2012). Itinerario terapéutico de las personas con discapacidad y mediaciones en el cuidado de la salud: la mirada de los familiares. Physis Revista de Saúde Coletiva, 22 (3), pp. 1063-1083. Doi: $\quad$ http://dx.doi.org/10.1590/S010373312012000300012. 